Pacific Journal of Mathematics

INTRINSIC EXTENSIONS OF RING 


\title{
INTRINSIC EXTENSIONS OF RINGS
}

\author{
CARL FAith AND YUZo Utumi
}

A module $M$ is an essential extension of a submodule $N$ in case $K \cap N \neq 0$ for each nonzero submodule $K$ of $M$. If $S$ is a subring of a ring $R$, and if ${ }_{S} R,{ }_{S} S$ denote the left $S$-modules naturally defined by the ring operations of $R$, then $R$ is a left quotient ring of $S$ in case ${ }_{S} R$ is an essential extension of ${ }_{S} S$.

We shall discuss the following problem: (1) Characterize the condition that a ring extension $R$ of $S$ is a left quotient ring of $S$ wholly in terms of the relative left ideal structures of $R$ and $S$.

A ring extension $R$ of $S$ is left intrinsic over $S$ in case $K \cap S \neq 0$ for each nonzero left ideal $K$ of $R$. Evidently each left quotient ring $R$ of $S$ is left intrinsic over $S$ but an obvious example (when $R$ is a field and $S$ a subfield $\neq R$ ) shows that the converse fails. Nevertheless, we ask: (2) When is the condition $R$ is left intrinsic over $S$ a solution to (1)?

We now specialize $S$ by requiring that:

(i) $S$ possesses a left quotient ring which is a (von Neumann) regular ring, or equivalently (R. E. Johnson [2]) by requiring that the left singular ideal of $S$ vanishes. For such a ring there exists a maximal left quotient ring $\widehat{S}$ which is unique up to isomorphism over $S$, and which is itself a regular ring ([2]). To eliminate the field example we require that:

(ii) $\widehat{S}$ possesses no strongly regular ideals $\neq 0$. Under these hypotheses we present the following solution to (1).

A. THEOREM (2.6). Let $S$ satisfy (i) and (ii). Then an extension ring $R$ of $S$ is a left quotient ring of $S$ if and only if $R$ is a left intrinsic extension of $S$ such that for each closed left ideal $A$ of $S$ there corresponds a left ideal $B$ of $R$ such that $B \cap S=A$.

(See $\S 1$ for definitions.)

Regarding (2) we add a rather dubious final hypothesis:

(iii) $\hat{S}$ is right intrinsic over $S$.

B. THEOREM (3.1). If $S$ satisfies (i)-(iii), then an extension ring $R$ of $S$ is a left quotient ring of $S$ if and only if $R$ is left

Received May 15, 1963. The first author gratefully acknowledges partial support from the National Science Foundation under grants G-19863 and G-21514. 
intrinsic over $S$. (Then there exists a ring monomorphism of $R$ into $\widehat{S}$ which is the identity on $S$.)

Combining $B$ with a theorem of Goldie [1] we obtain:

C. THEOREM (3.2). Let $S$ be a prime ring which is both left and right noetherian, and assume that $S$ is not an integral domain. Let $Q$ denote the classical quotient ring of $S$ ([1]). Then an extension ring $R$ of $S$ is left intrinsic over $S$ if and only if there exists a ring monomorphism of $R$ into $Q$ which is the identity on $S$.

1. A ring $S$ is strongly regular (resp. regular) if for any $x \in S$ there exists $y \in S$ such that $x^{2} y=x$ (resp. $x y x=x$ ); an ideal $I$ of $S$ is strongly regular if $I$ is a strongly regular ring.

Let $S$ be a ring. Then ${ }_{S} M$ will denote that $M$ is a left $S$-module, and ${ }_{S} S$ denotes the left $S$-module defined naturally by the ring operations in $S .{ }_{s} M$ is an essential extension of a submodule $N$ in case $K \cap N \neq 0$ for each submodule $K \neq 0$ of $M$. Then, $N$ is said to be an essential submodule of $M$. An essential left ideal of $S$ is a left ideal of $S$ which is an essential submodule of ${ }_{s} S$. (Thus a left ideal $I$ of $S$ is essential if and only if $S$ is a left intrinsic extension of $I_{\text {.) }}$ )

An element $x \in{ }_{s} M$ is singular in case the annihilator of $x$ in $S$ is an essential left ideal of $S$. It is known that the set $Z\left({ }_{S} M\right)$ of singular elements of ${ }_{S} M$ is a submodule of ${ }_{s} M$, called the singular submodule of $\left.{ }_{s} M ; Z{ }_{s} S\right)$ is an ideal of $S$ called the left singular ideal of $S$.

If $Z\left({ }_{S} S\right)=0$, then $S$ is said to be a $J_{l}$-ring, and $\hat{S}$ denotes its maximal left quotient ring; $\hat{S}$ is a regular ring with identity, and is left self-injective. If $R$ is any left self-injective ring with identity, then it is known (Utumi [4], Lemma 8) that $Z\left(_{R} R\right.$ ) coincides with the Jacobson radical $J(R)$ of $R$, and that the difference $R-J(R)$ is a regular ring.

A left ideal $A$ of a ring $S$ is closed if there is no left ideal of $S$ which is a proper essential extension of $A$.

In case $S$ is a $J_{l}$-ring it is known that the set of closed left ideals of $S$ forms a complete complemented modular lattice $L(S)$. If $R$ is a left quotient ring of $S, R$ is also a $J_{l}$-ring, and $L(R)$ is isomorphic to $L(S)$ by the correspondence $A(\in L(R)) \rightarrow A \cap S$. Thus, in this case, the following condition is satisfied:

(1.1) Condition. Let $R$ be an extension ring of a ring $S$. For any closed left ideal $A$ of $S$ there is a left ideal $B$ of $R$ such that $B \cap S=A$. 
We call an extension ring $R$ of $S$ left strongly intrinsic if $R$ is a left intrinsic extension of $S$, and if Condition 1.1 is fulfilled.

(1.2) Lemma. Let $R$ be a left intrinsic extension of a $J_{l}$-ring $S$. Suppose that the maximal left quotient ring of $S$ is right intrinsic over $S$. Then $R$ is a left strongly intrinsic extension of $S$.

Proof. Let $A$ be a closed left ideal of $S$. By [5, Theorem 2.2] $A$ is an annihilator left ideal of $S$. Hence $B \cap S=A$ for some annihilator left ideal $B$ of $R$, as desired.

It is evident that Condition 1.1 is equivalent to the following:

(1.3) If $A$ and $B$ are left ideals of $S$ such that $A \cap B=0$, then there exists a left ideal $C$ of $R$ such that $C \supset A$ and $C \cap B=0$.

Let $R$ be a left strongly intrinsic extension of a ring $S$. Then the following three properties are easily seen:

(1.4) If $A \cap B=0$ for a left ideal $A$ of $R$ and a left ideal $B$ of $S$, there is a left ideal $C$ of $R$ such that $A \cap C=0$.

(1.5) If the sum of left ideals $\left\{A_{i}\right\}$ of $S$ is direct, so is the sum of $\left\{A_{i}+R A_{i}\right\}$.

(1.6) If $A$ is an essential left ideal of $S$, then $A+R A$ is an essential left ideal of $R$. If $C$ is an essential left ideal of $R, C \cap S$ is also an essential left ideal of $S$.

(1.7) Lemma. Let $R$ be a left strongly intrinsic extension of a ring $S$. Then the following properties are equivalent:

(i) $S$ is a $J_{l}$-ring;

(ii) $R$ is a $J_{l}$-ring;

(iii) the singular submodule $Z\left(_{s} R\right)$ of the left $S$-module $R$ is zero.

Proof. By (1.6) it is obvious that $Z\left({ }_{s} R\right) \subset Z\left({ }_{R} R\right) \subset Z\left({ }_{s} R\right)$. Hence $Z\left({ }_{s} R\right)=Z\left({ }_{R} R\right)$. This shows the equivalence of (ii) and (iii). Now $Z\left({ }_{S} S\right)=Z\left({ }_{S} R\right) \cap S=Z\left({ }_{R} R\right) \cap S$. Since $Z\left({ }_{R} R\right)$ is an ideal of $R$, $Z\left({ }_{R} R\right)=0$ if and only if $Z\left({ }_{R} R\right) \cap S=0$. Therefore (i) is equivalent to (ii), as desired.

The following proposition is known:

(1.8) Let $S$ be a $J_{l}$-ring. A left ideal $A$ of $S$ is closed if and only if $B x \subset A$ for $x \in S$ and an essential left ideal $B$ of $S$ implies that $x \in A$. 
(1.9) Lemma. Let $R$ be a left strongly intrinsic extension of a $J_{l}$-ring $S$. Then the lattice $L(R)$ of closed left ideals of $R$ is isomorphic to the lattice $L(S)$ of closed left ideals of $S$ under contraction $A \rightarrow A \cap S$.

Proof. It is direct from (1.6) and (1.8) that $A \cap S$ is closed for any closed $A$. Suppose that $A_{1} \cap S=A_{2} \cap S$ for $A_{1}, A_{2} \in L(R)$. It is known that any intersection of closed left ideals of a $J_{l}$-ring is closed. Thus, $\left(A_{3}=\right) A_{1} \cap A_{2} \in L(R)$. Let $B$ be a left ideal of $R$ such that $B \subset A_{1}, B \cap A_{3}=0$. It follows then that $B \cap S=0$. Hence $B=0$, which shows that $A_{1}=A_{3}$, since $A_{3}$ is closed. Similarly $A_{2}=A_{3}$, and therefore $A_{1}=A_{2}$. Finally we shall show that the correspondence is onto. Let $C \in L(S)$. By (1.1), $C=D \cap S$ for some left ideal $D$ of $R$. By Zorn's lemma there exists a maximal left ideal $E$ of $R$ such that $E \cap S=C$. Let $F$ be a left ideal of $R$ which contains $E$ properly. Then $\mathrm{F} \cap S \neq C$. Since $C$ is closed, we can find a nonzero left ideal $G$ of $S$ such that $G \subset F \cap S$ and $G \cap C=0$. By (1.1) there is a left ideal $H$ of $R$ such that $H \cap S$ is an essential extension of $G$. Then $0=(H \cap S) \cap C=((H \cap F) \cap E) \cap S$. Since $R$ is left intrinsic over $S$, we have that $(H \cap F) \cap E=0$. This implies that $F$ is not an essential extension of $E$. Therefore $E \in L(R)$, completing the proof.

2. The following proposition is easily verified:

(2.1) Let $M$ be a left $S$-module with zero singular submodule. Suppose that a left ideal $A$ of $S$ is an essential extension of a left. ideal $B$ of $S$. Let $v$ and $w$ be left $S$-homomorphisms of $A$ into $M$. If $(v-w) B=0$, then $v=w$.

In fact, $v-w$ induces a homomorphism of $A / B$ into $M$. By assumption $Z(A / B)=A / B$. Hence $(v-w) A=Z((v-w) A) \subset Z(M)=0$, as desired.

A left $S$-module $M$ is called injective if for any left ideal $A$ of $S$, and for any left $S$-homomorphism $v$ of $A$ into $M$ there exists an element $x$ such that $v(a)=a x$ for every $a \in A$. A ring $S$ is called left self injective if the left $S$-module $S$ is injective, and $S$ has a unit element. Any left self injective ring which is semisimple (in the sense of Jacobson) is regular (in the sense of von Neumann). As. is known, the maximal left quotient ring of a $J_{l}$-ring is semisimple, in the sense of Jacobson, and left self injective.

We denote by $l(P, Q)$ the set of $x \in P$ with $x Q=0$. Similarly $r(P, Q)$ denotes the right annihilator, in $P$, of $Q$.

(2.2) Lemma. Let $R$ be a semisimple left self injective ring, and suppose that it is a left strongly intrinsic extension of a ringr 
S. Then the left S-module $R$ is an injective module with zero singular submodule.

Proof. Since $R$ is regular, it is a $J_{l}$-ring. By Lemma (1.7), $Z\left({ }_{S} R\right)=0$. Let $A$ be a left ideal of $S$, and $v$ a left $S$-homomorphism of $A$ into $R$. Denote the left ideal of $S$ generated by an element $x$ by $(x)_{l}$. By Zorn's lemma there is a maximal subset $\left\{x_{i}\right\}$ of $A$ such that the sum $B$ of $\left(x_{i}\right)_{l}$ is direct. Evidently $A$ is an essential extension of $B$. By (1.5), the sum of $\left\{S x_{i}\right\}$ is also direct. Now $l\left(S, x_{i}\right) \subset l\left(S, v\left(x_{i}\right)\right)$, that is, $l\left(R, x_{i}\right) \cap S \subset l(R, v(x)) \cap S$. Since any annihilator left ideal of the $J_{l}$-ring $R$ is closed, it follows by Lemma 1.9 that $l\left(R, x_{i}\right) \subset l\left(R, v\left(x_{i}\right)\right)$. This shows that $x_{i} \rightarrow v\left(x_{i}\right)$ generates a left $R$-homomorphism $w$ of $\sum_{i} R x_{i}$ into $R$. By the injectivity of ${ }_{R} R$ there exists an element $a \in R$ such that $v\left(x_{i}\right)=x_{i} a$ for every $i$. Thus the homomorphism $v$ and the right multiplication of $a$ coincide on $B$. Since $A$ is essential over $B$, it follows by (2.1) that $v$ is given by the right multiplication of $a$, completing the proof.

(2.3) THEOREM. Let $R$ be an extension ring of a ring $S$, and suppose that the left $S$-module $R$ is an injective module with zero singular submodule. Then $S$ is a $J_{l}$-ring. Let $T$ be the maximal one among such submodules of the left $S$-module $R$ that are essential over the left $S$-module $S$. Then $T$ forms a subring of $R$, and in fact it is the maximal left quotient ring of $S$.

Proof. Since $Z\left({ }_{S} S\right)=Z\left({ }_{S} R\right) \cap S=0, S$ is a $J_{l}$-ring. Let $E$ be the endomorphism ring of ${ }_{s} R$, and let $v \in E$. In case ${ }_{s} R$ is essential over $\left.\operatorname{Ker} v, Z{ }_{s} R / \operatorname{Ker} v\right)=R / \operatorname{Ker} v$, and so $\operatorname{Im} v=Z(\operatorname{Im} v) \subset Z(R)=$ 0 , whence $v=0$. In view of [4, Lemma 8] it follows from this that $E$ is semisimple, and $T$ is uniquely determined. Since ${ }_{s} T$ is essential over ${ }_{S} S$, it is easy to see that the set $D(x)$ of elements $y$ of $S$ such that $y x \in S$ is an essential left ideal of $S$ for each $x \in T$. Now we denote by $U$ the set of $x \in R$ such that $D(x)$ is an essential left ideal of $S$. Clearly $T \subset U$. It is not difficult to show that $U$ is a subring of $R$. Since $Z\left({ }_{s} R\right)=0,{ }_{s} U$ is essential over ${ }_{S} S$. Hence $U \subset T$, therefore $T=U$. Thus, $T$ forms a subring of $R$. $T$ is the maximal left quotient ring of $S$ because ${ }_{S} T$ is the maximal essential extension of ${ }_{S} S$.

(2.4) Lemma. Let $R$ be a ring with unit, and suppose that it is a left strongly intrinsic extension of a semisimple left self injective ring $S$. Then every idempotent of $R$ belongs to $S$.

Proof. Let $e=e^{2} \in R$. Then $R e \in L(R)$, the lattice of closed left ideals of $R$. Hence $R e \cap S \in L(S)$, the lattice of closed left ideals of 
$S$, by Lemma 1.9. Since every closed left ideal of $S$ is a principal left ideal generated by an idempotent, $S \cap R e=S f$ for some $f=$ $f^{2} \in S$. Evidently $R f \cap S=S f=R e \cap S$, and hence $R e=R f$ by Lemma 1.9. Similarly we can find an idempotent $g \in S$ such that $R(1-e)=R g$. Since $S$ is regular, $S f+S g=S h$ for some $h=h^{2} \in S$. Then $R h=R$, and $h=1$. Hence $1=x f+y g$ for some $x, y \in S$. Also,

$$
e-x f=e-(1-y g)=y g-(1-e) \in R g+R(1-e)=R(1-e) .
$$

Hence $e-x f \in R e \cap R(1-e)=0$, and therefore $e=x f \in S$, completing the proof.

By virtue of [5, Corollary to Theorem 4], any semisimple left self injective ring $R$ is decomposed into the direct sum of two ideals $R_{1}$ and $R_{2}$ in such a way that $R_{1}$ is strongly regular, and $R_{2}$ does not contain any nonzero strongly regular ideals. The decomposition is unique. By [5, Theorem 2], $R_{2}$ is generated by idempotents.

(2.5) THEOREM. Let $R$ be semisimple left self injective ring, and let $R=R_{1} \oplus R_{2}$ be the decomposition into ideals mentioned above. Suppose that $R$ is a left strongly intrinsic extension of a ring $S$. Then there is a subring $T$ of $R_{1}$ with the following properties:

(i) $T$ contains every idempotent of $R_{1}$,

(ii) $T$ is a strongly regular, (left) self injective ring and

(iii) $T \oplus R_{2}$ is the maximal left quotient ring of $S$.

Proof. By Lemma 2.2, ${ }_{s} R$ is injective and $Z\left({ }_{s} R\right)=0$. Thus, by Lemma 2.3, $R$ contains as a subring the maximal left quotient ring $Q$ of $S$. Since $R$ is left intrinsic over $S$, and $R \supset Q \supset S$, it is evident that $R$ is left intrinsic over $Q$. Let $A$ be a closed left ideal of $Q$. Then $A=Q e, e=e^{2} \in Q$, and hence $A=R e \cap Q$. This shows that $R$ is left strongly intrinsic over $Q$. Thus, by Lemma 2.4 every idempotent of $R$ belongs to $Q$. Since $R_{2}$ is generated by idempotents, $R_{2} \subset Q$, and so $Q=\left(Q \cap R_{1}\right) \oplus R_{2}$. Set $T=Q \cap R_{1}$. Since $Q$ is regular, so is its ideal $T$. Thus, the strong regularity of $T$ follows from the fact that a regular ring is strongly regular if and only if it has no nonzero nilpotent elements, completing the proof.

(2.6) THEOREM. Let $S$ be a $J_{l}$-ring. Suppose that the maximal left quotient ring of $S$ does not contain any nonzero strongly regular ideals. Then any left strongly intrinsic extension of $S$ is a left quotient ring of $S$.

Proof. Let $R$ be a left strongly intrinsic extension of $S$, and denote by $Q$ the maximal left quotient ring of $R$. By Lemma 1.9, 
$Q$ is a left strongly intrinsic extension of $S$. Let $Q=Q_{1} \oplus Q_{2}$ where $Q_{1}$ is a strongly regular ideal of $Q$, and $Q_{2}$ is an ideal of $Q$ which does not contain any nonzero strongly regular ideals. By Theorem 2.5 there is a strongly regular subring $T$ of $Q_{1}$ such that $T \oplus Q_{2}$ is the maximal left quotient ring of $S$. $T$ contains every idempotent of $Q_{1}$, especially the unit element of $Q_{1}$. Since $T=0$ by assumption, it follows that $Q_{1}=0$. Thus, $Q=Q_{2}$ is the maximal left quotient ring of $S$. Since $Q \supset R \supset S, R$ is a left quotient ring of $S$, as desired.

3. The following is the main theorem.

(3.1) THEOREM. Let $S$ be a $J_{l}$-ring. Suppose that the maximal left quotient ring of $S$ is right intrinsic, and does not contain any nonzero strongly regular ideals. Then any left intrinsic extension of $S$ is a left quotient ring.

Proof. By Lemma 1.2 any left intrinsic extension of $S$ is left strongly intrinsic over $S$, and hence it is a left quotient ring of $S$ by Theorem 2.6, as desired.

Goldie proved in [1; Theorem 13] that if a prime ring $S$ satisfies the maximum conditions for left and right ideals, then $S$ may be imbedded into a simple ring $R$ with minimum condition in such a way that $R$ is the classical quotient ring of $S$ in the following sense:

(i) Every non-zero-divisor of $S$ has the inverse in $R$;

(ii) every element $x$ of $R$ is of the forms $a^{-1} b$ and $c d^{-1}$ for some $a, b, c$ and $d \in S$. In this case $S$ is a $J_{l}$-ring, and $R$ is the maximal left quotient ring of $S$. Since $R$ is a right quotient ring of $S$, it is right intrinsic over $S$. $R$ contains a nonzero strongly regular ideal if and only if $R$ is a division ring, that is, $S$ is an Ore domain. Thus, by Theorem 3.1 we obtain the following.

3.2. THEOREM. Let $S$ be a prime ring with maximum conditions for left and right ideals, and suppose that it is not an Ore domain. Then an extension ring of $S$ is left (or right) intrinsic over $S$ if and only if it is isomorphic, over $S$, to a between ring of $S$ and the classical quotient ring of $S$.

\section{REFERENCES}

1. A. W. Goldie, The structure of prime rings under ascending chain conditions, Proc. London Math. Sec., (3) 8 (1958), 589-608.

2. R. E. Johnson, The extended centralizer of a ring over a module, Proc. Amer. Math. Soc., 2 (1951), 891-895.

3. Yuzo Utumi, On quotient rings, Osaka Math. J., 8 (1956), 1-18.

4. — On a theorem on modular lattices, Proc. Japan Acad., 35 (1959), 16-21. 
5. Yuzo, Utumi, On continuous regular rings and semisimple self injective rings, Can. J. Math., 12 (1960), 597-605.

6 . - On rings of which any one-sided quotient rings are two-sided, Proc. Amer. Math. Soc., 14 (1963), 141-147.

The Institute For AdvanCed Study and Rutgers UNIVERSity, THE UNIVERSITY OF ROCHESTER 


\title{
PACIFIC JOURNAL OF MATHEMATICS
}

\author{
EDITORS
}

\author{
Robert Osserman \\ Stanford University \\ Stanford, California \\ M. G. Arsove \\ University of Washington \\ Seattle 5, Washington
}

\author{
J. Dugundji \\ University of Southern California \\ Los Angeles 7, California \\ Lowell J. Paige \\ University of California \\ Los Angeles 24, California
}

\section{ASSOCIATE EDITORS}
E. F. BECKENBACH
B. H. NEUMANN
F. WOLF
K. YOSHIDA

\section{SUPPORTING INSTITUTIONS}

\author{
UNIVERSITY OF BRITISH COLUMBIA \\ CALIFORNIA INSTITUTE OF TECHNOLOGY \\ UNIVERSITY OF CALIFORNIA \\ MONTANA STATE UNIVERSITY \\ UNIVERSITY OF NEVADA \\ NEW MEXICO STATE UNIVERSITY \\ OREGON STATE UNIVERSITY \\ UNIVERSITY OF OREGON \\ OSAKA UNIVERSITY \\ UNIVERSITY OF SOUTHERN CALIFORNIA
}

\author{
STANFORD UNIVERSITY \\ UNIVERSITY OF TOKYO \\ UNIVERSITY OF UTAH \\ WASHINGTON STATE UNIVERSITY \\ UNIVERSITY OF WASHINGTON \\ AMERICAN MATHEMATICAL SOCIETY \\ CALIFORNIA RESEARCH CORPORATION \\ SPACE TECHNOLOGY LABORATORIES \\ NAVAL ORDNANCE TEST STATION
}

Mathematical papers intended for publication in the Pacific Journal of Mathematics should by typewritten (double spaced), and on submission, must be accompanied by a separate author's résumé. Manuscripts may be sent to any one of the four editors. All other communications to the editors should be addressed to the managing editor, L. J. Paige at the University of California, Los Angeles 24, California.

50 reprints per author of each article are furnished free of charge; additional copies may be obtained at cost in multiples of 50 .

The Pacific Journal of Mathematics is published quarterly, in March, June, September, and December. Effective with Volume 13 the price per volume (4 numbers) is $\$ 18.00$; single issues, $\$ 5.00$. Special price for current issues to individual faculty members of supporting institutions and to individual members of the American Mathematical Society: $\$ 8.00$ per volume; single issues $\$ 2.50$. Back numbers are available.

Subscriptions, orders for back numbers, and changes of address should be sent to Pacific Journal of Mathematics, 103 Highland Boulevard, Berkeley 8, California.

Printed at Kokusai Bunken Insatsusha (International Academic Printing Co., Ltd.), No. 6, 2-chome, Fujimi-cho, Chiyoda-ku, Tokyo, Japan.

PUBLISHED BY PACIFIC JOURNAL OF MATHEMATICS, A NON-PROFIT CORPORATION

The Supporting Institutions listed above contribute to the cost of publication of this Journal, but they are not owners or publishers and have no responsibility for its content or policies. 


\section{Pacific Journal of Mathematics}

\section{Vol. 14, No. $2 \quad$ June, 1964}

Tom M. (Mike) Apostol and Herbert S. Zuckerman, On the functional equation $F(m n) F((m, n))=F(m) F(n) f((m, n)) \ldots \ldots \ldots \ldots \ldots \ldots \ldots \ldots \ldots \ldots \ldots$

Reinhold Baer, Irreducible groups of automorphisms of abelian groups . . . . . . . 385

Herbert Stanley Bear, Jr., An abstract potential theory with continuous kernel . . . . 407

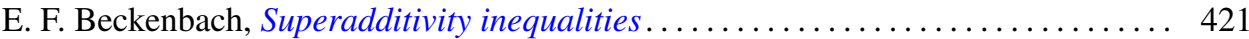

R. H. Bing, The simple connectivity of the sum of two disks . . . . . . . . . . . 439

Herbert Busemann, Length-preserving maps ...................... 457

Heron S. Collins, Characterizations of convolution semigroups of measures . . . . . . 479

Paul F. Conrad, The relationship between the radical of a lattice-ordered group and complete distributivity............................ 493

P. H. Doyle, III, A sufficient condition that an arc in $S^{n}$ be cellular . . . . . . . . . 501

Carl Clifton Faith and Yuzo Utumi, Intrinsic extensions of rings . . . . . . . . . . 505

Watson Bryan Fulks, An approximate Gauss mean value theorem . . . . . . . . . . 513

Arshag Berge Hajian, Strongly recurrent transformations . . . . . . . . . . . . . 517

Morisuke Hasumi and T. P. Srinivasan, Doubly invariant subspaces. II . . . . . . . 525

Lowell A. Hinrichs, Ivan Niven and Charles L. Vanden Eynden, Fields defined by

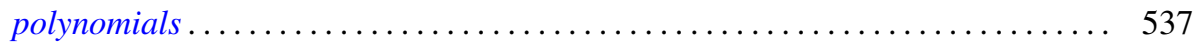

Walter Ball Laffer, I and Henry B. Mann, Decomposition of sets of group

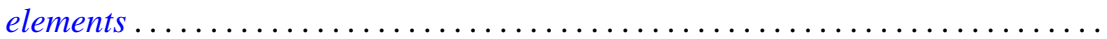

John Albert Lindberg, Jr., Algebraic extensions of commutative Banach

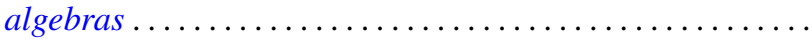

W. Ljunggren, On the Diophantine equation $C x^{2}+D=y^{n} \ldots$

M. Donald MacLaren, Atomic orthocomplemented lattices ....

Moshe Marcus, Transformations of domains in the plane and applications in the

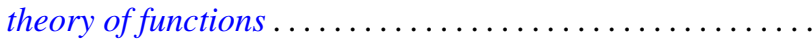

Philip Miles, $B^{*}$ algebra unit ball extremal points . .................. 627

W. F. Newns, On the difference and sum of a basic set of polynomials . . . . . . . 639

Barbara Osofsky, Rings all of whose finitely generated modules are injective ...... 645

Calvin R. Putnam, Toeplitz, matrices and invertibility of Hankel matrices . . . . . . . 651

Shoichiro Sakai, Weakly compact operators on operator algebras . . . . . . . . . 659

James E. Simpson, Nilpotency and spectral operators . . . . . . . . . . . . . 665

Walter Laws Smith, On the elementary renewal theorem for non-identically

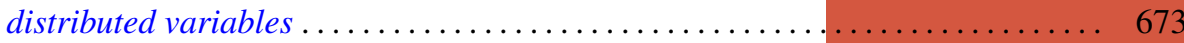

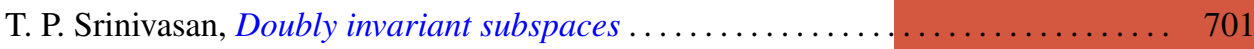

J. Roger Teller, On the extensions of lattice-ordered groups . . . . . . . . . . . . 709

Robert Charles Thompson, Unimodular group matrices with rational integers as

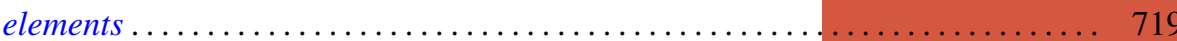

J. L. Walsh and Ambikeshwar Sharma, Least squares and interpolation in roots of unity

Charles Edward Watts, A Jordan-Hölder theorem .................... 731

Kung-Wei Yang, On some finite groups and their cohomology .............. 735

Adil Mohamed Yaqub, On the ring-logic character of certain rings ............ 741

Paul Ruel Young, A note on pseudo-creative sets and cylinders 